\title{
On the number of decomposable trees
}

\author{
Stephan G. Wagner $]^{\dagger}$ \\ Institut für Analysis und Computational Number Theory, Technische Universität Graz, Steyrergasse 30, 8010 Graz, \\ Austria
}

A tree is called $k$-decomposable if it has a spanning forest whose components are all of size $k$. Analogously, a tree is called $T$-decomposable for a fixed tree $T$ if it has a spanning forest whose components are all isomorphic to $T$. In this paper, we use a generating functions approach to derive exact and asymptotic results on the number of $k$-decomposable and $T$-decomposable trees from a so-called simply generated family of trees - we find that there is a surprisingly simple functional equation for the counting series of $k$-decomposable trees. In particular, we will study the limit case when $k$ goes to $\infty$. It turns out that the ratio of $k$-decomposable trees increases when $k$ becomes large.

Keywords: decomposable tree, simply generated family of trees, generating functions, asymptotic analysis

\section{Introduction}

Counting certain types of graphs or trees certainly ranges among the classical problems of combinatorics. A special class of trees for which several kinds of enumeration problems have been successfully treated is the class of so-called simply generated trees in the sense of Meir and Moon (cf. [9]). A simply generated family of trees is determined by a sequence $c_{0}=1, c_{1}, c_{2}, \ldots$ of weights. The weight of a rooted ordered tree is then given by

$$
c(T)=\prod c_{i}^{N_{i}(T)},
$$

where $N_{i}(T)$ is the number of vertices in $T$ with exactly $i$ children. Now one can define a generating function for the total weight of all trees on $n$ vertices via

$$
T(x)=\sum_{T} c(T) x^{|T|},
$$

satisfying a functional equation of the form $T(x)=x \Phi(T(x))$, where $\Phi(t)=\sum_{i=0}^{\infty} c_{i} t^{i}$. Special cases include rooted plane trees $\left(\Phi(t) \frac{1}{1-t}\right)$, rooted labelled trees $\left(\Phi(t)=e^{t}\right.$; in this case, the exponential generating function is used) and $s$-ary trees $\left(\Phi(t)=(1+t)^{s}\right)$; the following theorem provides information on the asymptotic behaviour of the coefficients of $T(x)$ :

Theorem 1 (Meir/Moon [9]) Suppose $\Phi(t)$ is an analytic function of $t$ for $|t|<R \leq \infty$, and let $T(x)=$ $\sum_{n \geq 0} t_{n} x^{n}$ denote the unique solution of $T(x)=x \Phi(T(x))$ in the neighborhood of $x=0$. If

- $c_{k} \geq 0$ for $k \geq 1$,

- $\operatorname{gcd}\left(k: c_{k}>0\right)=1$, and

- $\tau \Phi^{\prime}(\tau)=\Phi(\tau)$ for some $0<\tau<R$, then

$$
t_{n} \sim a \rho^{-n} n^{-3 / 2}
$$

as $n \rightarrow \infty$, where $\rho \tau / \Phi(\tau)$ and $a\left(\Phi(\tau) /\left(2 \pi \Phi^{\prime \prime}(\tau)\right)\right)^{1 / 2}$.

In a recent paper of Bell, Burris and Yeats [3], this result was extended to a very general theorem about families of rooted trees.

Graph decompositions, on the other hand, have been intensively studied in various forms and have many applications. Generally, an $n$-vertex graph $G=(V, E)$ is called $\lambda$-decomposable for some partition $\lambda=$

\footnotetext{
${ }^{\dagger}$ Supported by the Austrian Science Foundation FWF, project S9611, part of the Austrian National Research Network "Analytic Combinatorics and Probabilistic Number Theory".
} 
$\left(\lambda_{1}, \ldots, \lambda_{p}\right)$ of $n$ if there exists a partition $\left(V_{1}, \ldots, V_{p}\right)$ of $V$ such that the graph induced by $V_{i}$ is connected and $\left|V_{i}\right| \lambda_{i}(1 \leq i \leq p)$. The best-known special case is certainly given by the partition $\lambda(2, \ldots, 2)-\mathrm{a}$ perfect matching of a graph is just a $(2, \ldots, 2)$-decomposition.

Graph decomposition of the described form are even of interest in the theory of parallel computers and networks - given a network system, one wants to efficiently divide it into subsystems of given size and assign a subsystem to each application. For further information on this topic, we refer to [2] and the references therein. The question of characterizing $\lambda$-decomposable trees - from a theoretical as well as from an algorithmical point of view - is treated in several papers such as [1, 2, 8].

A natural generalization of the special case $\lambda(2, \ldots, 2)$ is given by $\lambda(k, \ldots, k)$. For the sake of brevity, we will call a $(k, \ldots, k)$-decomposable graph $k$-decomposable. An equivalent definition is the following:

Definition 1 A tree $T$ is called $k$-decomposable if there is a spanning forest of $T$ whose components all have size $k$.

A natural reason to consider the decomposability of trees is the fact that a connected graph is $\lambda$-decomposable if and only if it has a $\lambda$-decomposable spanning tree [2]. It has been shown by Zelinka [13] that the decomposition, if it exists, is always unique. 2-decomposable trees (equivalently, those with a perfect matching) were studied in several papers, such as [10, 11, 12]. In particular, Moon [10] investigates simply generated trees with a perfect matching and shows that the corresponding counting series $M(x)$ satisfies the functional equation

$$
M(x)=x^{2} \Phi(M(x)) \Phi^{\prime}(M(x)) .
$$

In this paper we are going to prove a generalization of this equation to $k$-decomposable trees. This will enable us to determine explicit formulas in certain special cases as well as asymptotic ones. Finally, we will discuss further possible variants of the concept of decomposability.

\section{Establishing functional equations}

Let $k$ be fixed and let $A(x) A_{0}(x)$ denote the generating function for the number of $k$-decomposable trees within a simply generated family of trees, given be the function $\Phi$. Furthermore, let $A_{l}(x)$ be the generating function for the number of trees which have a spanning forest such that all components have size $k$, except the component containing the root, which has size $l$. Let the class of trees with this property be denoted by $\mathcal{T}_{l}$.

Now, let $R$ be a rooted tree that belongs to $\mathcal{T}_{l}$. Then the subtrees $R_{1}, R_{2}, \ldots$ of $R$ have to belong to some $\mathcal{T}_{a_{i}}$ such that $a_{1}+a_{2}+\ldots=l-1$. Note that the number of subtrees belonging to $\mathcal{T}_{0}$ (which is supposed to denote the class of $k$-decomposable trees) is arbitrary. In terms of the generating functions, we have

$$
A_{l}(x)=x \sum_{r_{1}+2 r_{2}+\ldots=l-1} \frac{A_{1}(x)^{r_{1}} A_{2}(x)^{r_{2}} \cdots}{r_{1} ! r_{2} ! \ldots} \Phi^{\left(r_{1}+r_{2}+\ldots\right)}\left(A_{0}(x)\right),
$$

where $\Phi^{\left(r_{1}+r_{2}+\ldots\right)}$ is the $\left(r_{1}+r_{2}+\ldots\right)$-th derivative of $\Phi$. In this formula, $r_{i}$ stands for the number of subtrees of type $\mathcal{T}_{i}$. Writing $A(x, y)=\sum_{l \geq 1} A_{l}(x) y^{l}$, this equation translates to

$$
A(x, y)=x y \sum_{n \geq 0} \frac{\Phi^{(n)}\left(A_{0}(x)\right)}{n !} A(x, y)^{n} .
$$

Now, we can prove the following explicit formula:

\section{Proposition 2}

$$
A_{l}(x)=\frac{x^{l}}{l !}\left(\Phi^{l}\right)^{(l-1)}\left(A_{0}(x)\right) .
$$

Proof: By the Lagrange inversion formula (cf. [6]), we have

$$
\begin{aligned}
A_{l}(x)=\left[y^{l}\right] A(x, y) & =\frac{x^{l}}{l}\left[u^{l-1}\right]\left(\sum_{n \geq 0} \frac{\Phi^{(n)}\left(A_{0}(x)\right)}{n !} u^{n}\right)^{l} \\
& =\frac{x^{l}}{l} \sum_{r_{1}+r_{2}+\ldots+r_{l}=l-1} \prod_{i \geq 1} \frac{\Phi^{\left(r_{i}\right)}\left(A_{0}(x)\right)}{r_{i} !} .
\end{aligned}
$$


Now, we transform the sum by writing $s_{j}$ for the number of appearances of $j$ among the $r_{i}$ :

$$
\begin{aligned}
A_{l}(x) & =\frac{x^{l}}{l} \sum_{\substack{s_{1}+2 s_{2}+\ldots=l-1 \\
s_{0}+s_{1}+s_{2}+\ldots l}}\left(\begin{array}{c}
l \\
s_{0}, s_{1}, s_{2}, \ldots
\end{array}\right) \prod_{j \geq 0}\left(\frac{\Phi^{(j)}\left(A_{0}(x)\right)}{j !}\right)^{s_{j}} \\
& =x^{l} \sum_{s_{1}+2 s_{2}+\ldots=l-1} \frac{(l-1) !}{\left(l-s_{1}-s_{2}-\ldots\right) !} \Phi\left(A_{0}(x)\right)^{l-\left(s_{1}+s_{2}+\ldots\right)} \prod_{j \geq 1} \frac{1}{s_{j} !}\left(\frac{\Phi^{(j)}\left(A_{0}(x)\right)}{j !}\right)^{s_{j}} \\
& =\frac{x^{l}}{l !} \sum_{s_{1}+2 s_{2}+\ldots=l-1}(l-1) !(l)_{s_{1}+s_{2}+\ldots} \Phi\left(A_{0}(x)\right)^{l-\left(s_{1}+s_{2}+\ldots\right)} \prod_{j \geq 1} \frac{1}{s_{j} !}\left(\frac{\Phi^{(j)}\left(A_{0}(x)\right)}{j !}\right)^{s_{j}},
\end{aligned}
$$

which equals $\frac{x^{l}}{l !}\left(\Phi^{l}\right)^{(l-1)}\left(A_{0}(x)\right)$ by Faà di Bruno's formula.

Now, since $A_{k}(x)=A_{0}(x)=A(x)$, we have

$$
A(x)=\frac{x^{k}}{k !}\left(\Phi^{k}\right)^{(k-1)}(A(x))
$$

for the generating function $A(x)$ of $k$-decomposable trees. This functional equation is of the same type as the functional equation for simply generated families themselves.

\section{Exact formulas in some special cases}

In special cases, the Lagrange inversion formula enables us to derive closed formulas for the number of $k$-decomposable trees. We are going to prove the following theorem:

Theorem 3 Let $\Phi(t)=(1+c t)^{m}$, where $c$ and $m$ have the same sign. Then the counting series for the number of $k$-decomposable trees is given by

$$
A(x)=\sum_{n \geq 1} \frac{1}{n}\left(\frac{(k m)_{k-1}}{k !}\right)^{n}\left(\begin{array}{c}
(k m-k+1) n \\
n-1
\end{array}\right) c^{k n-1} x^{k n} .
$$

If $\Phi(t)=\exp (c t)$ for some $c>0$, the counting series is given by

$$
A(x)=\sum_{n \geq 1} \frac{1}{n ! k !^{n}}(c k)^{k n-1} n^{n-1} x^{k n} .
$$

Remark 1 The theorem includes the special cases of rooted plane trees $\left(\Phi(t)=(1-t)^{-1}\right)$, s-ary trees $\left(\Phi(t)=(1+t)^{s}\right)$ and rooted labelled trees $(\Phi(t)=\exp (t))$.

Proof: Obviously, we have

$$
\frac{d^{k-1}}{d t^{k-1}}(1+c t)^{k m}=c^{k-1}(k m)_{k-1}(1+c t)^{k m-k+1} .
$$

The Lagrange inversion formula thus shows us that

$$
\begin{aligned}
{\left[x^{k n}\right] A(x) } & =\frac{1}{n}\left[u^{n-1}\right]\left(\frac{c^{k-1}(k m)_{k-1}}{k !}\right)^{n}(1+c u)^{(k m-k+1) n} \\
& =\frac{1}{n}\left(\frac{(k m)_{k-1}}{k !}\right)^{n}\left(\begin{array}{c}
(k m-k+1) n \\
n-1
\end{array}\right) c^{k n-1}
\end{aligned}
$$

in the first case. Similarly,

and thus

$$
\frac{d^{k-1}}{d t^{k-1}} \exp (c k t)=(c k)^{k-1} \exp (c k t)
$$

$$
\begin{aligned}
{\left[x^{k n}\right] A(x) } & =\frac{1}{n}\left[u^{n-1}\right] \frac{(c k)^{(k-1) n}}{k !^{n}} \exp (c k n u) \\
& =\frac{1}{n ! k !^{n}}(c k)^{(k-1) n}(c k n)^{n-1}=\frac{1}{n ! k !^{!}}(c k)^{k n-1} n^{n-1}
\end{aligned}
$$

in the second case. 
Remark 2 For (rooted) labelled trees, one can derive the exact formula by a simple combinatorial argument: let $T$ be a $k$-decomposable tree, and let its decomposition into components of size $k$ be given. Then one can construct a new tree $T^{\prime}$ by contracting each component to a single vertex and connecting vertices in $T^{\prime}$ if there is an edge between the corresponding components in $T$.

Now, in order to construct a $k$-decomposable labelled tree on $k n$ vertices, one can choose the labels assigned to the components in $\frac{(k n) !}{n ! k^{n}}$ ways, $T^{\prime}$ in $n^{n-2}$ ways, the components themselves in $\left(k^{k-2}\right)^{n}$ ways, and the edges between the components in $\left(k^{2}\right)^{n-1}$ ways. Multiplying these quantities readily yields the formula for the number of labelled $k$-decomposable trees:

$$
\frac{(k n) !}{n ! k !^{n}} \cdot n^{n-2} \cdot\left(k^{k-2}\right)^{n} \cdot\left(k^{2}\right)^{n-1}=\frac{(k n) !}{n ! k !^{n}} \cdot n^{n-2} k^{k n-2} .
$$

\section{The limit case}

In Theorem 3 of the preceding section, one observes that the ratio between the number of $k$-decomposable trees and the total number of trees on $k n$ vertices is given by

$$
\frac{(k n) !(k m n-k n+n) !}{(k m n) ! n !}\left(\frac{(k m)_{k-1}}{k !}\right)^{n}
$$

in the first case, which is asymptotically equal to

$$
\sqrt{\frac{k m-k+1}{m}} \cdot\left(\frac{k^{k}(k m-k+1)^{k m-k+1}(k m) !}{k !(k m)^{k m}(k m-k+1) !}\right)^{n} .
$$

Note that, for $k \rightarrow \infty$, we have

$$
\left(\frac{k^{k}(k m-k+1)^{k m-k+1}(k m) !}{k !(k m)^{k m}(k m-k+1) !}\right)^{1 / k} \rightarrow 1
$$

so that the ratio of $k$-decomposable trees decreases more slowly for larger $k$. Less formally speaking, it is easier to decompose a tree into large pieces than into small pieces.

A similar observation holds for the second case of Theorem 3 . There, the ratio is given by

$$
\frac{(k n) !}{n ! k !^{n}} n^{-(k-1) n} \sim \sqrt{k}\left(\frac{k^{k}}{e^{k-1} k !}\right)^{n},
$$

and again we have

$$
\left(\frac{k^{k}}{e^{k-1} k !}\right)^{1 / k} \rightarrow 1
$$

It is a natural question to ask whether this is just a coincidence or a general property of simply generated families of trees. The following theorem gives an answer to this question.

Theorem 4 Let $\Phi$ be a function satisfying the conditions of Theorem 1] and suppose that $0<\tau, \tau_{k}<$ $R$ are solutions of $\tau \Phi^{\prime}(\tau) \Phi(\tau)$ and $\tau_{k}\left(\Phi^{k}\right)^{(k)}\left(\tau_{k}\right)=\left(\Phi^{k}\right)^{(k-1)}\left(\tau_{k}\right)$ respectively. Then the ratio of $k$ decomposable trees among $k n$-vertex trees is asymptotically given by

$$
\frac{a_{k n}}{t_{k n}} \sim b_{k} \rho_{k}^{-k n},
$$

where

$$
\rho_{k} \frac{\Phi^{\prime}(\tau)}{\left(\left(\Phi^{k}\right)^{(k)}\left(\tau_{k}\right) / k !\right)^{1 / k}}
$$

and

$$
b_{k}=\left(\frac{k^{3} \Phi^{\prime \prime}(\tau)\left(\Phi^{k}\right)^{(k-1)}\left(\tau_{k}\right)}{\Phi(\tau)\left(\Phi^{k}\right)^{(k+1)}\left(\tau_{k}\right)}\right)^{1 / 2} .
$$

Furthermore, we have $\lim _{k \rightarrow \infty} \rho_{k}=1$. 
Proof: Note that the functional equation

$$
A_{k}(x)=\frac{x^{k}}{k !}\left(\Phi^{k}\right)^{(k-1)}(A(x))
$$

is essentially of the form $T(x)=x \Psi(T(x))$ again, so the theorem of Meir and Moon applies. This readily yields the asymptotic formula.

Now, we only have to prove the limit for $k \rightarrow \infty$. For this purpose, we need the following lemma:

Lemma 5 There are constants $B_{1}, B_{2}>0$ depending only on $\Phi$ such that -for sufficiently large $k$ - the inequalities

$$
B_{1} c_{k-1}^{(k)} \leq c_{k+1}^{(k)}
$$

and

$$
B_{2}^{n-k+1} c_{k-1}^{(k)} \geq c_{n}^{(k)}(n \geq k-1)
$$

hold, where $c_{n}^{(k)}=\left[t^{n}\right] \Phi^{k}(t)$.

Proof of Lemma 5: Note that $c_{n}^{(k)}=\left[t^{n} x^{k}\right] \frac{1}{1-\Phi(t) x}$. It follows from general theorems on generating functions (see, for instance, [5, Theorem 2]) that the coefficients $\left[t^{n} x^{k}\right] \frac{1}{1-\Phi(t) x}$ asymptotically follow a normal distribution for $k \rightarrow \infty$. More precisely,

$$
c_{n}^{(k)} \sim \frac{1}{\sqrt{2 \pi k \sigma^{2}\left(h\left(\frac{n}{k}\right)\right)}} \cdot \frac{\Phi\left(h\left(\frac{n}{k}\right)\right)^{k}}{h\left(\frac{n}{k}\right)^{n}},
$$

where

$$
\begin{gathered}
\mu(z)=\frac{z \Phi^{\prime}(z)}{\Phi(z)}, \\
\sigma^{2}(z)=\frac{z^{2} \Phi^{\prime \prime}(z) \Phi(z)+z \Phi(z)^{\prime} \Phi(z)-z^{2} \Phi^{\prime}(z)^{2}}{\Phi(z)^{2}},
\end{gathered}
$$

and $h$ is the inverse function of $\mu$. $(6)$ holds uniformly for $\frac{n}{k} \in[\mu(a), \mu(b)]$, where $[a, b]$ is a compact subinterval of $(0, R)$ and $R$ is the radius of convergence of $\Phi$. Now, we see that

$$
\begin{aligned}
\frac{c_{k-1}^{(k)}}{c_{k+1}^{(k)}} & \sim h(1)^{2}\left(\frac{\Phi(h(1))-(\Phi \circ h)^{\prime}(1) \frac{1}{k}+O\left(\frac{1}{k^{2}}\right)}{\Phi(h(1))+(\Phi \circ h)^{\prime}(1) \frac{1}{k}+O\left(\frac{1}{k^{2}}\right)}\right)^{k}\left(\frac{h(1)+h^{\prime}(1) \frac{1}{k}+O\left(\frac{1}{k^{2}}\right)}{h(1)-h^{\prime}(1) \frac{1}{k}+O\left(\frac{1}{k^{2}}\right)}\right)^{k} \\
& \sim h(1)^{2} \exp \left(-\frac{2(\Phi \circ h)^{\prime}(1)}{\Phi(h(1))}+\frac{2 h^{\prime}(1)}{h(1)}\right)=h(1)^{2}
\end{aligned}
$$

tends to a limit, which proves the first inequality of the lemma. Note at this point that $h(1)=\tau$ exists by the conditions of Theorem 1 and satisfies $\Phi^{\prime}(h(1)) \frac{\Phi(h(1))}{h(1)}$. The second inequality follows in a similar way.

Now, we can continue with the proof of the theorem. First of all, we have

$$
\tau_{k}\left(\Phi^{k}\right)^{(k)}\left(\tau_{k}\right)\left(\Phi^{k}\right)^{(k-1)}\left(\tau_{k}\right)
$$

and equivalently

$$
\sum_{n \geq k} c_{n}^{(k)}(n)_{k} \tau_{k}^{n-k+1}=\sum_{n \geq k-1} c_{n}^{(k)}(n)_{k-1} \tau_{k}^{n-k+1}
$$

or

$$
(k-1) ! c_{k-1}^{(k)}=\sum_{n \geq k+1} c_{n}^{(k)}(n-k)(n)_{k-1} \tau_{k}^{n-k+1} .
$$

Next, we observe that, by removing all summands with $n>k+1$,

$$
(k-1) ! c_{k-1}^{(k)} \geq \frac{(k+1) !}{2} c_{k+1}^{(k)} \tau_{k}^{2}
$$


and by Lemma 5

$$
\tau_{k} \leq \sqrt{\frac{2}{B_{1} k(k+1)}},
$$

so $\tau_{k} \ll \frac{1}{k}$. On the other hand, the second inequality of Lemma 5 shows that

$$
\begin{aligned}
c_{k-1}^{(k)} & \leq \sum_{n \geq k+1} c_{k-1}^{(k)}(n-k)\left(\begin{array}{c}
n \\
k-1
\end{array}\right)\left(B_{2} \tau_{k}\right)^{n-k+1} \\
& =c_{k-1}^{(k)}\left(1+\left(1-B_{2} \tau_{k}\right)^{-(k+1)}\left((k+1) B_{2} \tau_{k}-1\right)\right),
\end{aligned}
$$

which means that $\tau_{k} \geq \frac{1}{B_{2}(k+1)} \gg \frac{1}{k}$.

Clearly,

$$
\begin{aligned}
\frac{1}{k !}\left(\Phi^{k}\right)^{(k)}\left(\tau_{k}\right) & \frac{1}{k ! \tau_{k}}\left(\Phi^{k}\right)^{(k-1)}\left(\tau_{k}\right) \\
& =\frac{1}{k ! \tau_{k}} \sum_{n \geq k-1} c_{n}^{(k)}(n)_{k-1} \tau_{k}^{n-k+1} \\
& \geq \frac{1}{k ! \tau_{k}} c_{k-1}^{(k)}(k-1) !=\frac{c_{k-1}^{(k)}}{k \tau_{k}}
\end{aligned}
$$

On the other hand,

$$
\begin{aligned}
\frac{1}{k !}\left(\Phi^{k}\right)^{(k)}\left(\tau_{k}\right) & \leq \frac{1}{k ! \tau_{k}} \sum_{n \geq k-1} c_{k-1}^{(k)}(n)_{k-1}\left(B_{2} \tau_{k}\right)^{n-k+1} \\
& =\frac{c_{k-1}^{(k)}}{k \tau_{k}} \sum_{n \geq k-1}\left(\begin{array}{c}
n \\
k-1
\end{array}\right)\left(B_{2} \tau_{k}\right)^{n-k+1} \\
& =\frac{c_{k-1}^{(k)}}{k \tau_{k}}\left(1-B_{2} \tau_{k}\right)^{-k}
\end{aligned}
$$

and the last factor is bounded since $\tau_{k} \ll \frac{1}{k}$.

Now, if $T(x)=x \Phi(T(x))$, the Lagrange inversion formula tells us that

$$
\left[x^{k}\right] T(x)=\frac{1}{k} c_{k-1}^{(k)} \sim a k^{-3 / 2} \Phi^{\prime}(\tau)^{k},
$$

from which the theorem follows easily.

\section{T-decomposability}

In this section, we impose a stronger condition on the decomposition of trees:

Definition 2 A tree is called T-decomposable for a fixed tree $T$ if it has a spanning forest whose components are all isomorphic to $T$.

If $|T|=k$, then, trivially, all $T$-decomposable trees are also $k$-decomposable, but not vice versa. In this paper, we will only investigate two special cases of $T$-decomposability in detail, namely the cases when $T=S_{k}$ is a $k$-vertex star or $T=P_{k}$ is a $k$-vertex path $(k \geq 3)$.

Let $S(x)$ be the counting series for $S_{k}$-decomposable trees. Again, we have to define certain auxiliary functions, viz. $S_{1}(x)$ and $S_{2}(x)$, which denote the counting series for the class of $S_{k}$-decomposable trees where the root is the center resp. a leaf of the star containing the root in the decomposition. Furthermore, $R(x)$ counts the class of rooted trees with the property that all subtrees are $S_{k}$-decomposable. It is not difficult to obtain the following system of functional equations:

$$
\begin{aligned}
S_{1}(x) & =x \frac{R(x)^{k-1}}{(k-1) !} \Phi^{(k-1)}(S(x)), \\
S_{2}(x) & =x^{2} \frac{R(x)^{k-2}}{(k-2) !} \Phi^{(k-2)}(S(x)) \Phi^{\prime}(S(x)), \\
R(x) & =x \Phi(S(x))
\end{aligned}
$$


and, trivially, $S_{1}(x)+S_{2}(x)=S(x)$. After some simple manipulations, we arrive at

$$
S(x)=\frac{x^{k}}{(k-1) !}\left(\Phi(S(x))^{k-1} \Phi^{(k-1)}(S(x))+(k-1) \Phi(S(x))^{k-2} \Phi^{(k-2)}(S(x)) \Phi^{\prime}(S(x))\right) .
$$

Again, it is possible to derive explicit formulas in some special cases:

Theorem 6 Let $\Phi(t)=(1+c t)^{m}$, where $c$ and $m$ have the same sign. Then the counting series for the number of $S_{k}$-decomposable trees is given by

$$
S(x)=\sum_{n \geq 1} \frac{1}{n}\left(\frac{(m)_{k-2}(k m-k+2)}{(k-1) !}\right)^{n}\left(\begin{array}{c}
(k m-k+1) n \\
n-1
\end{array}\right) c^{k n-1} x^{k n} .
$$

If $\Phi(t)=\exp (c t)$ for some $c>0$, the counting series is given by

$$
S(x)=\sum_{n \geq 1} \frac{n^{n-1}}{k n !}\left(\frac{k^{2}}{(k-1) !}\right)^{n} c^{k n-1} x^{k n} .
$$

Note that the counting series coincide with those for $k$-decomposable trees in the case $k=3$. An asymptotic formula analogous to (5) also holds for $S_{k}$-decomposable trees.

Finally, let us consider $P_{k}$-decomposable trees. Denote by $P(x)$ the counting series for $P_{k}$-decomposable trees, and by $Q_{j}(x)$ the counting series for trees with the property that they can be decomposed into a collection of $P_{k}$ 's and a path $P_{j}$ starting in the root. Then it is not difficult to see that

$$
Q_{j}(x)=x Q_{j-1}(x) \Phi^{\prime}(P(x)) x^{j} \Phi(P(x)) \Phi^{\prime}(P(x))^{j-1} .
$$

Now, we divide the class of $P_{k}$-decomposable trees into two subclasses - those trees for which the root is an inner vertex resp. a leaf of the $P_{k}$ containing the root in the decomposition. Then, we derive the following functional equation:

$$
\begin{aligned}
P(x) & =x \sum_{l=1}^{k-2} Q_{l}(x) Q_{k-1-l}(x) \cdot \frac{\Phi^{\prime \prime}(P(x))}{2}+Q_{k} \\
& =x \sum_{l=1}^{k-2} x^{k-1} \Phi(P(x))^{2} \Phi^{\prime}(P(x))^{k-3} \cdot \frac{\Phi^{\prime \prime}(P(x))}{2}+x^{k} \Phi(P(x)) \Phi^{\prime}(P(x))^{k-1} \\
& =x^{k} \Phi^{\prime}(P(x))^{k-3}\left(\frac{k-2}{2} \Phi(P(x))^{2} \Phi^{\prime \prime}(P(x))+\Phi(P(x)) \Phi^{\prime}(P(x))^{2}\right) .
\end{aligned}
$$

We obtain the following exact formulas:

Theorem 7 Let $\Phi(t)=(1+c t)^{m}$, where $c$ and $m$ have the same sign. Then the counting series for the number of $P_{k}$-decomposable trees is given by

$$
P(x)=\sum_{n \geq 1} \frac{1}{n}\left(\frac{m^{k-2}(k m-k+2)}{2}\right)^{n}\left(\begin{array}{c}
(k m-k+1) n \\
n-1
\end{array}\right) c^{k n-1} x^{k n} .
$$

If $\Phi(t)=\exp (c t)$ for some $c>0$, the counting series is given by

$$
P(x)=\sum_{n \geq 1} \frac{n^{n-1}}{k n !}\left(\frac{k^{2}}{2}\right)^{n} c^{k n-1} x^{k n} .
$$

Note that the coefficients differ only by factors of $\left(\frac{m^{k-2}(k-1) !}{2(m)_{k-2}}\right)^{n}$ resp. $\left(\frac{(k-1) !}{2}\right)^{n}$ from those for $S_{k^{-}}$ decomposable trees, and that $\frac{m^{k-2}(k-1) !}{2(m)_{k-2}}$ tends to $\frac{(k-1) !}{2}$ as $m \rightarrow \infty$.

Remark 3 Again, a combinatorial argument can be used to obtain a formula for T-decomposable labelled trees. If $|T| k$ and $\lambda(T)$ denotes the number of labellings of $T$ (it is well known that $\lambda(T)=k !|\operatorname{Aut} T|^{-1}$ ), we see that the number of $T$-decomposable labelled trees is

$$
\frac{(k n) !}{n ! k ! n} \cdot n^{n-2} k^{2 n-2} \lambda(T)^{n} .
$$




\section{Open problems}

We conclude this paper by proposing some open problems which seem to be very natural in the context of decomposability:

- It is not far-fetched to suspect that Theorem 4 also holds for families of trees which are not simply generated, such as rooted (unordered) trees and free trees. However, a proof of this conjecture has to involve some new ideas.

- Can one give a closed formula for the number of $T$-decomposable trees on $n$ vertices in terms of $T$ and $n$ for other families of trees than the labelled one?

- Can one characterize the trees $T$ of given size for which the number of $T$-decomposable trees from some given class becomes minimal/maximal? In the labelled case, the final remark of the preceding section shows that the extremal trees are the star resp. all identity trees (trees whose isomorphism group is the identity).

\section{References}

[1] D. Barth, O. Baudon, and J. Puech, Decomposable trees: a polynomial algorithm for tripodes, Discrete Appl. Math. 119 (2002), no. 3, 205-216.

[2] Dominique Barth and Hervé Fournier, A degree bound on decomposable trees, Preprint available at www.prism.uvsq.fr/ hefou/publis/trees.pdf.

[3] Jason P. Bell, Stanley N. Burris, and Karen A. Yeats, Counting rooted trees: The universal law $t(n) c \rho^{-n} n^{-3 / 2}$, arXiv:math.CO/0512432.

[4] Reinhard Diestel, Graph theory, second ed., Graduate Texts in Mathematics, vol. 173, SpringerVerlag, New York, 2000.

[5] Michael Drmota, Asymptotic distributions and a multivariate Darboux method in enumeration problems, J. Combin. Theory Ser. A 67 (1994), no. 2, 169-184.

[6] I. P. Goulden and D. M. Jackson, Combinatorial enumeration, A Wiley-Interscience Publication, John Wiley \& Sons Inc., New York, 1983, With a foreword by Gian-Carlo Rota, Wiley-Interscience Series in Discrete Mathematics.

[7] Frank Harary and Edgar M. Palmer, Graphical enumeration, Academic Press, New York, 1973.

[8] Mirko Horňák and Mariusz Woźniak, Arbitrarily vertex decomposable trees are of maximum degree at most six, Opuscula Math. (2003), no. 23, 49-62.

[9] A. Meir and J. W. Moon, On the altitude of nodes in random trees, Canad. J. Math. 30 (1978), no. 5, 997-1015.

[10] J. W. Moon, The number of trees with a 1-factor, Discrete Math. 63 (1987), no. 1, 27-37.

[11] Rodica Simion, Trees with a 1-factor: degree distribution, Proceedings of the fifteenth Southeastern conference on combinatorics, graph theory and computing (Baton Rouge, La., 1984), vol. 45, 1984, pp. 147-159.

[13] Bohdan Zelinka, Partitionability of trees, Czechoslovak Math. J. 38(113) (1988), no. 4, 677-681. 